\title{
Two alternatives for disjunction: an inquisitive reconciliation*
}

\author{
Floris Roelofsen
}

January 27,2016

\begin{abstract}
There are two prominent semantic treatments of disjunction in formal semantics. Traditionally, disjunction is taken to express an operator that applies to any two elements $A$ and $B$ of a Boolean algebra and yields their join. In particular, if $A$ and $B$ are propositions, then disjunction delivers their union, $A \cup B$. Another, more recent proposal is to treat disjunction as expressing an operator that can apply to any two objects of the same semantic type, and yields the set consisting of these two objects. In particular, if disjunction applies to two propositions $A$ and $B$, it delivers a set of propositional alternatives, $\{A, B\}$. Each of the two approaches has certain merits that the other one lacks. Thus, it would be desirable to reconcile the two, combining their respective strengths. This paper shows that this is indeed possible, if we adopt a notion of meaning that does not just take truth-conditional, informative content into consideration, but also inquisitive content.
\end{abstract}

\section{Introduction}

There is a long tradition in formal semantics that analyzes disjunction words like English or as expressing an operator which, when applied to two elements $A$ and $B$ of a Boolean algebra, yields their join, that is, their least upper bound w.r.t. the partial order that the algebra is based on. In particular, if $A$ and $B$ are propositions, ordered by entailment, then disjunction delivers their union, $A \cup B$. An important virtue of this analysis is that it provides a uniform account of disjunction words across different languages and across different syntactic categories (Gazdar, 1980; Partee and Rooth, 1983; Keenan and Faltz, 1985). Moreover, it provides an explanation of the very fact that such words exist in virtually all languages: since the join operator is one of the most basic algebraic operations that can be performed on the elements of any Boolean algebra, and since the meanings of expressions of the relevant syntactic categories constitute such Boolean algebras, it is to be expected that languages generally have words to express the join operation on such meanings; just like it is to be expected, for instance, that many languages have words to express basic operations on quantities, like addition and subtraction.

However, there is also a substantial and rapidly growing body of work which promotes a different semantic treatment of disjunction, namely as generating sets of alternatives. In particular, when

\footnotetext{
${ }^{*}$ This paper has been presented, in chronological order, at Stanford, the University of Rochester, the University of Massachusetts Amherst, University College London, the University of Stuttgart, the University of Amsterdam, and the Third Questions in Discourse Workshop in Berlin. I received a lot of useful feedback on these occasions, for which I am very grateful. I am especially grateful to Maria Aloni, Ivano Ciardelli, Donka Farkas, and Jeroen Groenendijk; the paper has benefited enormously from our discussions and joint work over the last couple of years. Finally, financial support from the Netherlands Organisation for Scientific Research (NWO) is gratefully acknowledged.
} 
applied to two propositions $A$ and $B$, disjunction is taken to generate a set of two propositional alternatives, $\{A, B\}$. This treatment has been fruitfully applied to account for a number of phenomena, including disjunctive questions (e.g., von Stechow, 1991; Roelofsen and van Gool, 2010; AnderBois, 2011; Biezma and Rawlins, 2012), free choice effects arising from disjunction under possibility modals and in imperatives (e.g., Aloni, 2002, 2007b; Simons, 2005a,b; Aloni and Ciardelli, 2013), counterfactuals with disjunctive antecedents (e.g., Alonso-Ovalle, 2006; Champollion et al., 2015), unconditionals with disjunctive adjuncts (Rawlins, 2013), and sluicing with disjunctive antecedents (AnderBois, 2014). However, this alternative account of disjunction lacks the algebraic underpinning that made the traditional account so attractive. This makes it a less explanatory account: it is only motivated by the empirical observations that it is designed to account for, not by considerations that are independent of these observations.

Given that both approaches have their own merits, one would like to know whether it is possible to reconcile the two, combining their respective strengths. The general opinion in the literature seems to be that this is impossible. The treatment of disjunction as generating sets of alternatives has been presented as a real alternative for the traditional treatment, not as a refinement or an extension thereof. For instance, Alonso-Ovalle (2006) writes in the conclusion section of his dissertation:

"This dissertation has investigated the interpretation of counterfactuals with disjunctive antecedents, unembedded disjunctions, and disjunctions under the scope of modals. We have seen that capturing the natural interpretation of these constructions proves to be challenging if the standard analysis of disjunction, under which or is the Boolean join, is assumed."

Similarly, Simons (2005a) starts her paper as follows:

"In this paper, the meanings of sentences containing the word or and a modal verb are used to arrive at a novel account of the meaning of or coordinations. It has long been known that such sentences [...] pose a problem for the standard treatment of or as a Boolean connective equivalent to set union."

The aim of this paper is to show that the two approaches can be reconciled after all. What this requires, however, is that we move from the traditional notion of meaning, which only takes truth-conditional, informative content into consideration, to a more fine-grained notion that also takes inquisitive content into account, as proposed in recent work on inquisitive semantics (e.g., Ciardelli, Groenendijk, and Roelofsen, 2013, 2015). If we enrich our notion of meaning to encompass both informative and inquisitive content, entailment also becomes sensitive to these two aspects of meaning. What we will show is that, if disjunction is treated as a join operator with respect to this more fine-grained notion of entailment, it automatically generates the desired alternatives. Thus, we can have our cake and eat it: disjunction can be treated as a join operator and as introducing sets of alternatives at the same time - in inquisitive semantics the two go hand in hand.

The paper is organized as follows. Section 2 reviews the traditional treatment of disjunction as a join operator, highlighting what makes this treatment particularly attractive from a linguistic perspective. Section 3 reviews the treatment of disjunction as generating sets of alternatives, and briefly reviews a number of phenomena to which this treatment has been successfully applied. Section 4 shows how the two approaches can be reconciled, and Section 5 concludes. 
Before getting started, two remarks about the intended scope of the paper are in order. First, we will largely restrict our attention to sentential disjunction. The resulting treatment of sentential disjunction can be generalized to a cross-categorial account, but there are no particular novelties in that part of the proposal: the generalization works exactly the same way as in the traditional account. The main contribution of the paper is a treatment of sentential disjunction that incorporates both the rigorous algebraic underpinning of the traditional approach and the alternative generating behavior assumed in the more recent analyses.

Second, it should be emphasized that the main aim of the paper is a theoretical one, i.e., to show that two prominent semantic analyses of disjunction, which are generally thought of as competing analyses, can in fact be reconciled in a principled way. The aim is not to critically assess the empirical evidence that has been given in favor of these semantic analyses, let alone to provide any new empirical evidence for or against them. In this regard, it should be noted that some of the phenomena that have been dealt with by treating disjunction semantically as introducing sets of propositional alternatives - in particular free choice effects in modal constructions, as well as counterfactuals with disjunctive antecedents - have also received various other treatments. Some of these rely on a different non-standard semantic treatment of disjunction (e.g., Zimmermann, 2000; Geurts, 2005); others place the explanatory burden on pragmatics rather than invoking a non-standard semantics for disjunction (see, e.g. Schulz, 2005; Aloni, 2007a; Fox, 2007; Klinedinst, 2009; Chemla, 2009; Franke, 2009; van Rooij, 2010). A detailed comparison between the different accounts of these particular empirical phenomena is beyond the scope of the present paper. The contribution of the paper to this debate is just to show that one important objection against the propositional alternatives approach - namely that it forces us to give up the advantages of the traditional algebraic treatment of disjunction - can in fact be overcome.

Finally, note that in some other empirical domains - in particular that of disjunctive questionsthere is a rather broad consensus that a treatment of disjunction as generating sets of propositional alternatives is indispensable. So, even if this treatment of disjunction may not be strictly needed to account for modal free choice effects and counterfactuals with disjunctive antecedents, it is still well worth taking into serious consideration and trying to place on firmer theoretical ground.

\section{The traditional treatment of disjunction}

The meaning of a sentence - the proposition that it expresses - is standardly construed as a set of possible worlds, namely, those worlds in which the sentence is true. Thus, the proposition expressed by a sentence standardly embodies its truth conditions. As such, a proposition can be thought of as carving out a region in the space of all possible worlds. In asserting a sentence, a speaker is taken to provide the information that the actual world is located within this region, i.e., that the actual world is one in which the uttered sentence is true. Thus, the proposition expressed by a sentence captures the informative content of that sentence, i.e., the information that is provided by a speaker in uttering the sentence.

This means that we can compare two propositions in terms of their informative strength. One proposition is more informative than another if it carves out a smaller region, i.e., if it locates the actual world more precisely. Formally, we say that one proposition $A$ entails another proposition $B$, notation $A=B$, just in case $A \subseteq B$.

Entailment forms a partial order over the set of all propositions. Every partially ordered set has a certain algebraic structure and comes with certain basic algebraic operations. The set of 


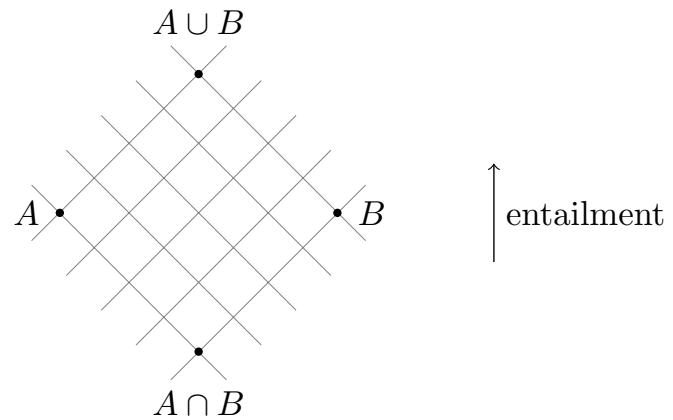

Figure 1: Join and meet.

propositions in standard truth-conditional semantics, ordered by entailment, forms a so-called Boolean algebra, which comes with three basic operations: join, meet, and complementation.

As depicted in Figure 1, the join of two propositions $A$ and $B$ is the least upper bound of $A$ and $B$ with respect to entailment, i.e., the strongest proposition that is entailed by both $A$ and $B$. This least upper bound amounts to the union of the two propositions: $A \cup B$.

The meet of $A$ and $B$ on the other hand, is the greatest lower bound of $A$ and $B$ with respect to entailment, i.e., the weakest proposition that entails both $A$ and $B$. This greatest lower bound amounts to the intersection of the two propositions: $A \cap B$.

The complement of a proposition $A$, which we denote as $A^{*}$, is the weakest proposition $C$ such that $A \cap C$ entails any other proposition. The only proposition that entails any other proposition is the empty proposition, $\emptyset$. Therefore, $A^{*}$ can be characterized as the weakest proposition $C$ such that $A \cap C=\emptyset$. It consists simply of all worlds that are not in $A$ itself: $A^{*}=\{w \mid w \notin A\}$.

Now, the standard treatment of disjunction, conjunction, and negation consists in associating each of these connectives with one of the basic algebraic operations: disjunction is taken to express the join operator, conjunction is taken to express the meet operator, and negation is taken to express complementation.

$$
\begin{aligned}
& \llbracket \varphi \vee \psi \rrbracket=\llbracket \varphi \rrbracket \cup \llbracket \psi \rrbracket \quad \text { join } \\
& \llbracket \varphi \wedge \psi \rrbracket=\llbracket \varphi \rrbracket \cap \llbracket \psi \rrbracket \quad \text { meet } \\
& \llbracket \neg \varphi \rrbracket=\llbracket \varphi \rrbracket^{*} \quad \text { complementation }
\end{aligned}
$$

Some simple propositions are depicted in Figure 2. In these examples, it is assumed that there are just two elementary sentences, $p$ and $q$, which means that there are just four distinct possible worlds: one where both $p$ and $q$ are true, labeled 11 , one where $p$ is true but $q$ is false, labeled 10, et cetera. Figure 2(a) depicts the proposition expressed by $p$, denoted $\llbracket p \rrbracket$, which is the set of all worlds where $p$ is true, and Figure 2(b) does the same for the proposition expressed by $q$, denoted $\llbracket q \rrbracket$. Figure 4(a) depicts the proposition expressed by $p \vee q$, which is the join of $\llbracket p \rrbracket$ and $\llbracket q \rrbracket$. Figure 2(d) depicts the proposition expressed by $p \wedge q$, which is the meet of $\llbracket p \rrbracket$ and $\llbracket q \rrbracket$. And finally, Figure 2(e) depicts the proposition expressed by $\neg p$, which is the complement of $\llbracket p \rrbracket$.

As mentioned above, since these algebraic operations are so basic, it is to be expected that natural languages will generally have lexical items that can be used to express them. And indeed, 


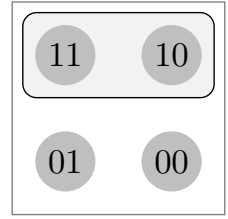

(a) $\llbracket p \rrbracket$

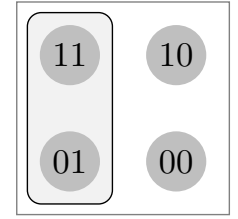

(b) $\llbracket q \rrbracket$

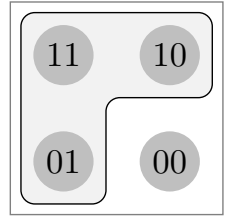

(c) $\llbracket p \vee q \rrbracket$

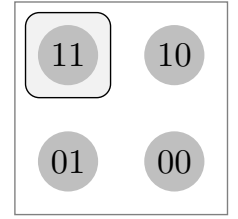

(d) $\llbracket p \wedge q \rrbracket$

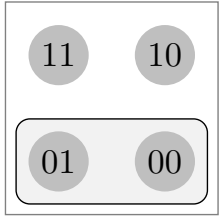

(e) $\llbracket \neg p \rrbracket$

Figure 2: Propositions expressed by some simple sentences in standard truth-conditional semantics.

in virtually all languages words or morphemes have been found that may be taken to fulfil exactly this purpose (see, e.g., Haspelmath, 2007). The algebraic perspective provides an explanation for this finding. Moreover, the account leads us to expect that disjunction, conjunction, and negation are not only applicable to full sentences, but also to expressions of other syntactic categories whose denotations form a suitable algebra, and that in this case they express exactly the same operations. This is again generally found to be the case across languages.

Note that the algebraic account is an explanatory account in that it is motivated by considerations that are independent from empirical observations concerning the behavior of connectives in natural languages. This makes the account particularly attractive, and indeed, it has for a long time been the standard. However, in relatively recent work, the treatment of disjunction as a join operator has been challenged, and an alternative account has been proposed. ${ }^{1}$ To this we turn next.

\section{Disjunction in alternative semantics}

\subsection{Alternative semantics}

On the alternative account (Simons, 2005a; Alonso-Ovalle, 2006; Aloni, 2007b, among others), sentential disjunction does not yield the join of the two propositions to which it applies, but rather generates a set containing these two propositions. For instance, as depicted in Figure $3, \llbracket p \vee q \rrbracket$ is not taken to amount to $\llbracket p \rrbracket \cup \llbracket q \rrbracket$, but rather to $\{\llbracket p \rrbracket, \llbracket q \rrbracket\}$.

The treatment of disjunction as introducing sets of alternatives is part of a broader line of work, which analyzes various other kinds of constructions as introducing sets of alternatives as well, including wh-words (Hamblin, 1973), focus (Rooth, 1985), indeterminate pronouns (e.g., Kratzer and Shimoyama, 2002) and indefinites (e.g., Kratzer, 2005; Menéndez-Benito, 2005; Aloni, 2007b). The general framework that has emerged from this work is referred to as alternative semantics.

As mentioned in the introduction, the treatment of disjunction proposed in alternative semantics has been fruitfully applied to a number of empirical phenomena, involving questions, imperatives, modals, conditionals, unconditionals, and sluicing. To illustrate the approach, let us briefly review two of these applications. ${ }^{2}$

\footnotetext{
${ }^{1}$ The algebraic treatment of conjunction has also been subject to critical debate. In particular, it has been debated whether the word and can be treated as a meet operator in sentences involving collective predication, like John and Sue got married (see Champollion, 2015, for a recent overview and defense of the algebraic approach).

${ }^{2}$ These brief illustrations are not intended to provide an overview of the literature on the phenomena at hand, let alone to argue for a specific account of them. They are just meant to give a sense for why the treatment of disjunction as generating multiple propositional alternatives has been found useful in these empirical domains.
} 


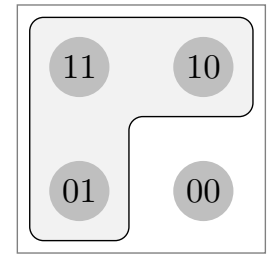

(a) standard

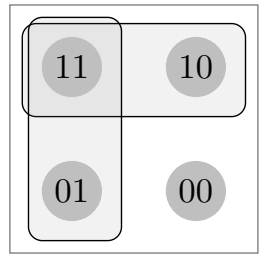

(b) alternative

Figure 3: $\llbracket p \vee q \rrbracket$ in standard truth-conditional semantics and in alternative semantics.

\subsection{Some illustrations}

\section{Disjunctive questions}

A disjunctive question like (1) can be pronounced in various ways, resulting in different interpretations (von Stechow, 1991; Bartels, 1999; Han and Romero, 2004; Roelofsen and van Gool, 2010; Biezma and Rawlins, 2012; Pruitt and Roelofsen, 2013; Roelofsen and Farkas, 2015, among others).

Does Igor speak English or French?

If pronounced with a prosodic phrase break after the first disjunct, pitch accents on both disjuncts, and falling intonation on the second disjunct, (1) is interpreted as an alternative question. In this case, it presupposes that Igor speaks exactly one of the two languages and elicits a response that establishes which of the two languages it is.

If pronounced without a prosodic phrase break after the first disjunct, without a pitch accent on the first disjunct, and with rising intonation on the second disjunct, (1) is interpreted as a polar question. In this case, it does not presuppose that Igor speaks one of the two languages; rather, it elicits a response that establishes whether this holds or not. That is, the issue that the question expresses with this intonation pattern can be resolved either by establishing that Igor speaks one of the two languages (without necessarily specifying which of the two), or by establishing that he doesn't speak either of them.

Finally, if pronounced with a prosodic phrase break after the first disjunct, pitch accents on both disjuncts, and rising intonation on both disjuncts, (1) is interpreted as an open question. In this case, it does not presuppose that Igor speaks one of the two languages, just like in the polar question interpretation; however, the issue that it expresses with this intonation pattern is different, namely one that can be resolved either by establishing that Igor speaks English, or by establishing that he speaks French, or by establishing that he doesn't speak either of the two (establishing that 


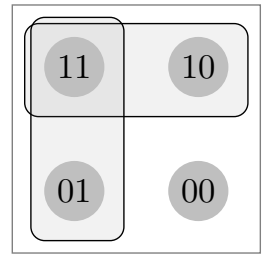

(a) $p \vee q$

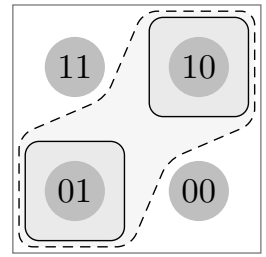

(b) $Q_{\text {alt }}(p \vee q)$

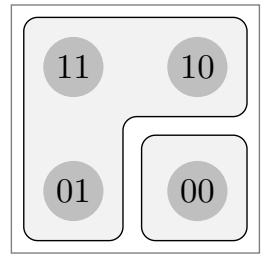

(c) $Q_{\text {polar }}(p \vee q)$

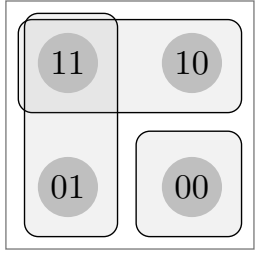

(d) $Q_{\text {open }}(p \vee q)$

Figure 4: A bare disjunction $p \vee q$ and three types of disjunctive questions formed out of it. Translation key: $p$ stands for 'Igor speaks English', $q$ for 'Igor speaks French, 11 is a world where both are true, 10 a world where only $p$ is true, et cetera.

he speaks one of the two languages without specifying which of the two is not sufficient). ${ }^{3,4}$

These different readings of disjunctive questions can be derived if we assume that disjunction generates a set of propositional alternatives; in our example, there are two such alternatives, as depicted in Figure 4(a). One alternative consists of all worlds where Igor speaks English, the other consists of all worlds where Igor speaks French.

On a simplistic account, each intonation pattern may be taken to reflect the presence of a different question operator: $Q_{\text {alt }}, Q_{\text {polar }}$, and $Q_{\text {open }}$, respectively. These operators may then be taken to apply to the two alternatives generated by the disjunction, each with a different output.

As depicted in Figure 4(b), we may assume that $Q_{\text {alt }}$ strengthens both alternatives in such a way that they become mutually exclusive (removing the overlap between them), and that it further adds a presupposition that one of these strengthened alternatives holds (this presupposition is depicted in Figure 4(b) with dashed borders).

On the other hand, as depicted in Figure 4(c), we may assume that $Q_{\text {polar }}$ 'merges' the two alternatives generated by the disjunction into one big alternative, consisting of all worlds where Igor speaks at least one of the two languages, and then adds the complement of this alternative, consisting of all worlds where Igor does not speak either of the two languages.

Finally, as depicted in Figure $4(\mathrm{~d})$, we may assume that $Q_{\text {open }}$ does not merge the two alternatives generated by the disjunction, and does not make them mutually exclusive either, but leaves them untouched and just adds the set of worlds where Igor does not speak either of the two languages as a third alternative.

Of course, while this simplistic account derives the three different readings, the real challenge is to unravel how the various syntactic and intonational properties of each question type conspire to

\footnotetext{
${ }^{3}$ This third reading is not as often considered in the literature as the first two; people often find it difficult to distinguish it from the polar question reading if the context of utterance is left unspecified. A context in which the open question reading of (1) is very prominent is the following: the speaker wants to write a letter to Igor, a distant relative from Russia who she has never met before; the addressee does know Igor pretty well; the speaker of course wants to write to Igor in a language that they both speak; the speaker doesn't speak any Russian, so she is listing some languages that she does speak; since she doesn't know much about Igor she cannot presuppose that he does indeed speak one of these languages, let alone that he speaks exactly one of them. Note that a plain Yes response to an open question is not satisfactory, though Yes, English or Yes, French are fine, and No is also fine (see Roelofsen and van Gool, 2010; Pruitt and Roelofsen, 2011; Roelofsen and Farkas, 2015, for further discussion and an account).

${ }^{4}$ The three intonation patterns are described here at a rather superficial level. For more elaborate discussion and some experimental results concerning the precise connection between the possible interpretations of disjunctive questions and their possible intonation patterns, see Bartels (1999) and Pruitt and Roelofsen (2013).
} 
bring these readings about. This challenge has been addressed in the literature in much more depth than can be done justice to here (see, e.g., von Stechow, 1991; Aloni and van Rooij, 2002; Han and Romero, 2004; Beck and Kim, 2006; Aloni and Égré, 2010; Haida, 2010; Roelofsen and van Gool, 2010; Pruitt and Roelofsen, 2011; AnderBois, 2011; Biezma and Rawlins, 2012; Roelofsen, 2013b; Uegaki, 2014; Roelofsen and Farkas, 2015). Our purpose here is just to illustrate why the treatment of disjunction as generating sets of propositional alternatives has been particularly successful in this empirical domain: what is crucial is that this treatment makes it possible for operators that scope over a disjunction - here, the various $Q$ operators - to access the alternatives contributed by each individual disjunct. This would not be possible on the traditional treatment of disjunction, where the semantic value of a disjunctive clause is a single proposition, without any trace of what was contributed by each individual disjunct (compare Figures 3(a) and 3(b)).

\section{Counterfactuals with disjunctive antecedents}

Consider the following scenario. Sally had a birthday party at her house, and some friends brought their instruments. One of Sally's friends, Bart Balloon, who has a terrible musical taste, fortunately forgot to bring his trumpet. Now consider the following sentence:

(2) If Bart Balloon had played the trumpet, people would have left.

$$
\varphi>\psi
$$

According to the classical treatment of counterfactuals due to Stalnaker (1968) and Lewis (1973), $\varphi>\psi$ is true in a world $w$ just in case, among all worlds that make $\varphi$ true, those that differ minimally from $w$ also make $\psi$ true. This correctly predicts that (2) is true in the given scenario.

But now consider a counterfactual with a disjunctive antecedent:

If Bart Balloon or Louis Armstrong had played the trumpet, people would have left.

The Stalnaker/Lewis treatment of counterfactuals, together with the classical treatment of disjunction, wrongly predicts that (3) is true in the given scenario as well. This is because all worlds that make the antecedent true and differ minimally from the actual world are ones where Bart Balloon played the trumpet, and not Louis Armstrong. The second disjunct in the antecedent is thus effectively disregarded.

Initially, this observation was presented as an argument against the Stalnaker/Lewis treatment of counterfactuals (Fine, 1975; Nute, 1975; Ellis et al., 1977; Warmbrōd, 1981). But another way to approach the problem is to pursue an alternative treatment of disjunction (Alonso-Ovalle, 2006, 2009; van Rooij, 2006; Fine, 2012; Champollion et al., 2015). Indeed, if the disjunctive antecedent is taken to generate two propositional alternatives and if verifying the counterfactual involves separately supposing each of the alternatives generated by the antecedent, the problem is avoided.

Thus, just like in the case of disjunctive questions, what makes the treatment of disjunction as generating multiple propositional alternatives attractive here is that it allows operators that scope over a disjunction - in this case the counterfactual - to access the alternatives contributed by each individual disjunct, something that would be impossible on the traditional account. ${ }^{5}$

\footnotetext{
${ }^{5}$ As already anticipated in the introduction, there are also approaches to the puzzle presented by counterfactuals with disjunctive antecedents that place the entire explanatory burden on pragmatics rather than invoking a nonstandard semantic treatment of disjunction (e.g., Chemla, 2009; Franke, 2009; Klinedinst, 2009; van Rooij, 2010). A critical assessment of the different approaches to the puzzle would of course be interesting in its own right, but is beyond the scope of the present paper.
} 


\subsection{Impasse}

We have just given two brief illustrations of phenomena which have been fruitfully analyzed in alternative semantics. As mentioned above, the full range of such phenomena is much broader. However, while the treatment of disjunction as generating multiple alternatives has been successful in terms of empirical coverage, it has limited explanatory power. After all, unlike the traditional algebraic account, it has not been motivated by considerations that are independent from the empirical observations that it is intended to capture.

Thus, we seem to have reached an impasse. Both semantic treatments of disjunction have their own merits, but the two approaches rest on different, seemingly incompatible assumptions. Ideally we would be able to bring the two closer together and obtain an analysis that shares their respective virtues. But how could this be achieved?

\section{Reconciliation}

I will show that the two approaches can be reconciled after all. In order to make this possible, however, a rather fundamental step needs to be taken. Namely, we need to move from the traditional truth-conditional notion of meaning, which captures only informative content, to a more general notion of meaning that encompasses both informative and inquisitive content. Such a generalized notion of meaning has been developed in recent work on inquisitive semantics (e.g., Ciardelli, Groenendijk, and Roelofsen, 2013, 2015). This work is rooted in the seminal work of Hamblin (1973), Karttunen (1977), and Groenendijk and Stokhof (1984) on the semantics of questions. However, it involves some innovations that are essential for our purposes here. Most importantly, inquisitive semantics comes with a well-behaved notion of entailment, which is sensitive to both informative and inquisitive content. This is crucial to preserve the algebraic treatment of the connectives. ${ }^{6}$ Indeed, disjunction can be treated in this framework as a join operator, just like in standard truth-conditional semantics, but now with respect to a more fine-grained notion of entailment. And indeed, if we do this, disjunction automatically generates the desired alternatives. Let us see how this works in more detail.

\subsection{Propositions in inquisitive semantics}

We start by recalling some standard notions. Let $W$ be the set of all possible worlds. Each possible world encodes a possible way the world may be. A set of possible worlds $s \subseteq W$ can be thought of as encoding a certain body of information, namely the information that the actual world corresponds to one of the elements of $s$. In particular, $s$ can be taken to encode the information state of one of the conversational participants, or the body of information that is shared among the conversational participants at a given point. Following Stalnaker (1978), we will refer to the latter body of information as the common ground of the conversation.

\footnotetext{
${ }^{6}$ The lack of an appropriate notion of entailment was one of the main criticisms that Groenendijk and Stokhof (1984) brought up against Hamblin (1973), Karttunen (1977), and other early work on the semantics of questions. Groenendijk and Stokhof's partition framework partly overcomes this problem; however, as noted by Groenendijk and Stokhof themselves, while the resulting notion of entailment yields a suitable treatment of conjunction as a meet operator, it does not yield an appropriate treatment of disjunction and the other connectives. Inquisitive semantics can be seen as a generalization of the partition framework, which fully regains the traditional algebraic treatment of the connectives (see Roelofsen, 2013a; Ciardelli, 2014, for more elaborate discussion).
} 
In standard truth-conditional semantics, the proposition expressed by a sentence is also construed as a set of possible worlds. As remarked above, in this framework a proposition intuitively carves out a region in the space of all possible worlds, and in asserting a sentence, a speaker is taken to provide the information that the actual world is located within this region. In this way, the proposition expressed by the sentence captures the informative content of the sentence.

Now, if we want propositions to be able to capture both informative and inquisitive content, i.e., both the information that is provided and the issue that is raised by a speaker in uttering a sentence, they cannot simply be construed as sets of possible worlds. However, there is a very natural generalization of the traditional notion of propositions that does suit this purpose. Namely, propositions can be construed as sets of information states. In uttering a sentence, a speaker can then be taken to steer the common ground of the conversation towards one of the states in the proposition expressed, while at the same time providing the information that the actual world must be contained in one of these states.

The proposition expressed by a sentence thus encodes a proposal to enhance the common ground in one or more ways. If the common ground is enhanced in one of these ways, we say that the proposal is settled in the resulting information state. Furthermore, we assume that the proposal remains settled if the resulting state is further enhanced. That is, the set of information states in which a proposal is settled is downward closed: if a proposal is settled in a state $s$, then it remains settled in any state $s^{\prime} \subseteq s$. In particular, as a limit case, any proposal is taken to be settled in the inconsistent state $\emptyset$.

A proposition is taken to consist of all information states that settle the proposal that it encodes. This means that not just any set of information states constitutes a proposition. In order for this to be the case, the set has to be downward closed, and it has to contain the inconsistent state. Equivalently, it has to be downward closed and non-empty. These considerations lead to the following notion of propositions in inquisitive semantics: ${ }^{7}$

Definition 1 (Propositions in inquisitive semantics).

A proposition in inquisitive semantics is a non-empty, downward closed set of information states.

Among the states that settle a proposition $A$, the ones that are easiest to reach are those that contain least information. The states in $A$ that contain least information are those that contain most possible worlds, i.e., they are the maximal elements of $A$. These maximal elements are refered to in inquisitive semantics as the alternatives in $A$. In depicting a proposition, we will generally only depict the alternatives that it contains.

Finally, since in uttering a sentence that expresses a proposition $A$ a speaker is taken to provide the information that the actual world is contained in one of the elements of $A$, i.e., in $\bigcup A$, we refer to $\bigcup A$ as the informative content of $A$, and denote it as info $(A)$.

To illustrate these notions, consider the following two sentences:

Did Amy leave?

(5) Amy left.

\footnotetext{
${ }^{7}$ It is sometimes found confusing at first sight that the term 'proposition' does not refer to sets of possible worlds in inquisitive semantics, as it does in truth-conditional semantics, but rather to sets of information states. To appreciate this terminological choice it helps to recognize that, even though propositions in inquisitive semantics are different kinds of objects than in truth-conditional semantics, they play exactly the same role as they do in the traditional framework. To use Frege's familiar distinction, the term refers to different kinds of entities in the two frameworks, but its sense is kept constant.
} 


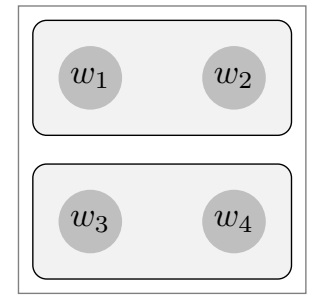

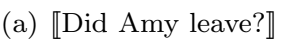

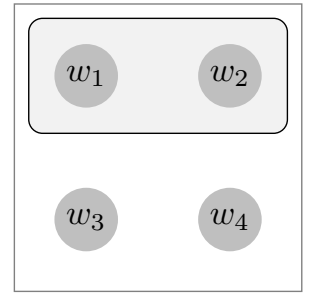

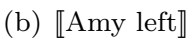

Figure 5: Some simple propositions in inquisitive semantics.

These sentences may be taken to express the propositions depicted in Figure 5, where $w_{1}$ and $w_{2}$ are worlds where Amy left, $w_{3}$ and $w_{4}$ are worlds where Amy didn't leave, and the shaded rectangles are the alternatives contained in the given propositions (by downward closure, all substates of these alternatives are also contained in the given propositions).

The proposition in Figure 5(a) captures the fact that in uttering the polar question Did Amy leave?, a speaker (i) provides the trivial information that the actual world must be $w_{1}, w_{2}, w_{3}$, or $w_{4}$ (all options are open) and (ii) steers the common ground towards a state that is either within $\left\{w_{1}, w_{2}\right\}$, where it is known that Amy left, or within $\left\{w_{3}, w_{4}\right\}$, where it is known that Amy didn't leave. Other conversational participants need provide information in order to establish such a common ground.

On the other hand, the proposition in Figure 5(b) captures the fact that in uttering the declarative Amy left, a speaker (i) provides the information that the actual world must be either $w_{1}$ or $w_{2}$, i.e., one where Amy left, and (ii) steers the common ground of the conversation towards a state in which it is commonly accepted that Amy left. In order to reach such a common ground, it is sufficient for other participants to accept the information that the speaker herself already provided in uttering the sentence; no further information is needed.

Notice that in the case of the polar question Did Amy leave?, the information that is provided is trivial in the sense that it does not exclude any possible world as a candidate for the actual world. A proposition $A$ is called informative in case it excludes at least one world, i.e., iff $\operatorname{info}(A) \neq W$.

On the other hand, in the case of the declarative Amy left, the inquisitive component of the proposition expressed is trivial, in the sense that in order to reach a state where the proposition is settled, other conversational participants only need to accept the informative content of the proposition expressed, and no further information is required. A proposition $A$ is called inquisitive just in case settling $A$ requires more than just mutual acceptance of $\operatorname{info}(A)$, i.e., iff $\operatorname{info}(A) \notin A$.

Given a picture of a proposition it is easy to see whether it is inquisitive or not. This is because, under the assumption that there are only finitely many possible worlds - and this is a safe assumption to make for all the examples to be considered in this paper - a proposition is inquisitive just in case it contains at least two alternatives. For instance, the proposition in Figure 5(a) contains two alternatives, which means that it is inquisitive, while the proposition in Figure 5(b) contains only one alternative, which means that it is not inquisitive. 


\subsection{Entailment and algebraic operations}

In order for one proposition $A$ to entail another proposition $B$ in inquisitive semantics, two conditions need to be fulfilled: (i) $A$ has to be at least as informative as $B$, and (ii) $A$ has to be at least as inquisitive as $B$. Whether the first condition is satisfied can be checked by comparing $\operatorname{info}(A)$ to $\operatorname{info}(B)$. Just as in truth-conditional semantics, $A$ is at least as informative as $B$ iff $\operatorname{info}(A) \subseteq \operatorname{info}(B)$. But what does it mean for $A$ to be at least as inquisitive as $B$ ? This can be made precise by comparing what it takes to settle $A$ with what it takes to settle $B$. More specifically, $A$ is at least as inquisitive as $B$ just in case every state that settles $A$ also settles $B$. But this just means that $A \subseteq B$. And moreover, if $A \subseteq B$, then it automatically holds that $\operatorname{info}(A) \subseteq \operatorname{info}(B)$ as well. Thus, at a formal level entailment in inquisitive semantics just amounts to inclusion, exactly as in standard truth-conditional semantics.

Definition 2 (Entailment in inquisitive semantics). $A \models B$ iff $A \subseteq B$

At this point we are back on familiar ground: we have a generalized notion of propositions and a corresponding generalized notion of entailment which induces a partial order on the set of all propositions. The next thing to do is to consider the algebraic structure of this partially ordered set, and the basic algebraic operations that can be performed on propositions in this setting.

What we find is that in inquisitive semantics, the set of propositions ordered by entailment forms a so-called Heyting algebra (Roelofsen, 2013a). This is a slight generalization of the Boolean algebra that we have in truth-conditional semantics. That is, Boolean algebras are a specific kind of Heyting algebras, ones where complements have a special property. Namely, while in all Heyting algebras it holds for any element $A$ that $A \cap A^{*}=\perp$, where $\perp$ is the strongest element of the algebra, in Boolean algebras it also holds for any element $A$ that $A \cup A^{*}=\top$, where $\top$ is the weakest element of the algebra. While this difference in algebraic structure is of course interesting in its own right, and has particular consequences for the behavior of negation in inquisitive semantics, what is most relevant for our purposes here is that any two propositions in inquisitive semantics still have a join and a meet, i.e., a least upper bound and a greatest lower bound w.r.t. entailment. Moreover, the join and the meet of two propositions can still be constructed simply by taking their union and intersection, respectively, just like in standard truth-conditional semantics.

Below we provide a proof of the fact that any two propositions in inquisitive semantics have a join, and that this join amounts to the union of these two propositions. For a proof of the analogous claim concerning meets and more elaborate formal discussion of the algebraic structure of the space of propositions in inquisitive semantics, we refer to Roelofsen (2013a).

Fact 1 (Joins). Any two propositions $A$ and $B$ in inquisitive semantics have a join, i.e., a least upper bound w.r.t. entailment, and this join amounts to $A \cup B$.

Proof. First, note that if $A$ and $B$ are propositions, then $A \cup B$ is also guaranteed to be a proposition, i.e., non-empty and downward closed. To see the latter, suppose that $s \in A \cup B$ and $s^{\prime} \subseteq s$. Then either $s \in A$ or $s \in B$. Without loss of generality, we may assume that $s \in A$. But then, since $A$ is downward closed, $s^{\prime} \in A$ as well, and therefore $s^{\prime} \in A \cup B$. So $A \cup B$ is downward closed.

Next, note that $A=A \cup B$ and $B=A \cup B$, which means that $A \cup B$ is an upper bound of $A$ and $B$ w.r.t. entailment. What remains to be shown is that $A \cup B$ is the least upper bound of $A$ and $B$. That is, for every proposition $C$ that is an upper bound of $A$ and $B$, we must show that $A \cup B=C$. To see this let $C$ be an upper bound of $A$ and $B$, and $s$ a state in $A \cup B$. Then $s$ must either be in $A$ or in $B$. Without loss of generality we may assume that $s \in A$. But then, since 


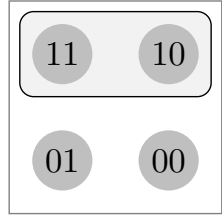

(a) $\llbracket p \rrbracket$

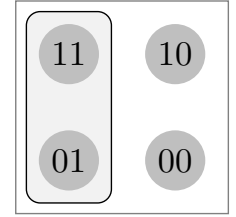

(b) $\llbracket q \rrbracket$

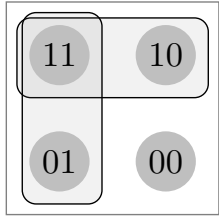

(c) $\llbracket p \vee q \rrbracket$

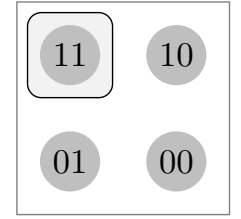

(d) $\llbracket p \wedge q \rrbracket$

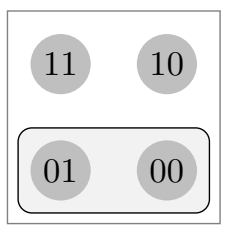

(e) $\llbracket \neg p \rrbracket$

Figure 6: Propositions expressed by some simple sentences in inquisitive semantics.

$A \models C, s$ must also be in $C$. This establishes that $A \cup B \models C$, which is what we set out to show. Thus, $A \cup B$ is indeed the least upper bound of $A$ and $B$.

Just like in truth-conditional semantics, then, disjunction can be taken to express the join operator, and similarly conjunction and negation can be taken to express the meet operator and complementation, respectively. Thus, while the notion of meaning has been enriched, the essence of the standard treatment of the connectives has been preserved.

$$
\begin{aligned}
& \llbracket \varphi \vee \psi \rrbracket=\llbracket \varphi \rrbracket \cup \llbracket \psi \rrbracket \quad \text { join } \\
& \llbracket \varphi \wedge \psi \rrbracket=\llbracket \varphi \rrbracket \cap \llbracket \psi \rrbracket \quad \text { meet } \\
& \llbracket \neg \varphi \rrbracket=\llbracket \varphi \rrbracket^{*} \quad \text { complementation }
\end{aligned}
$$

Let us look at a number of concrete examples to see how the algebraic operators behave in the inquisitive setting. For easy comparison, let us consider the simple sentences that we also considered above when discussing standard truth-conditional semantics (see Figure 2). The propositions expressed by these sentences in inquisitive semantics are depicted in Figure 6 .

The proposition expressed by an atomic sentence $p$ is the set of all states consisting of worlds where $p$ is true: $\llbracket p \rrbracket=\{s \mid \forall w \in s: w(p)=1\}$. This proposition contains a unique alternative, i.e., a unique maximal element, which is the set of all worlds where $p$ is true, $\{11,10\}$. This is depicted in Figure 6(a). Similarly, the proposition expressed by $q$ is the set of all states consisting of worlds where $q$ is true. Again, this proposition contains a unique alternative, which is the set of all worlds where $q$ is true, $\{11,01\}$. This is depicted in Figure 6(b).

Now consider the proposition expressed by $p \vee q$, depicted in Figure 6(c). This proposition contains two alternatives, precisely those associated with $p \vee q$ in alternative semantics, cf. Figure 3(b). Thus, while disjunction is treated as a join operator, just like in standard truth-conditional semantics, it also has the alternative-generating behavior that is assumed in alternative semantics.

Finally, the propositions expressed by $p \wedge q$ and by $\neg p$, depicted in Figures 6(d) and 6(e), respectively, both contain a single alternative, corresponding to the propositions expressed by these sentence in truth-conditional semantics.

\section{Concluding remarks}

Our aim has been accomplished: the two competing analyses of disjunction have been reconciled. This means that the empirical phenomena involving disjunction that have been successfully dealt with in alternative semantics can be accounted for without giving up the idea that disjunction expresses one of the most basic algebraic operations on meanings. In short, the obtained account 
unites the empirical advantages of alternative semantics with the explanatory merits of the traditional algebraic account.

There are a number of ways in which the basic result obtained here can be further extended. I will briefly provide some pointers to recent work where such extensions have been pursued.

Disjunction across syntactic categories. First, while we have restricted our attention to sentential disjunction in this paper, an important property of disjunction words in natural languages that was mentioned in the introduction is that they typically apply to expressions of many other syntactic categories as well. An important feature of the traditional treatment of the sentential connectives is that it can be generalized in a principled way to apply to expressions of all suitable categories (Gazdar, 1980; Partee and Rooth, 1983; Keenan and Faltz, 1985). This generalization can also be carried out in inquisitive semantics, in essentially the same way. This is done in detail in Theiler (2014); Ciardelli and Roelofsen (2015a), as part of a more general enterprise to develop a full-fledged type-theoretic inquisitive semantics.

Disjunction across clause types. Second, while we have focused in this paper on disjunction as such, the interpretation of sentences containing disjunction in natural languages of course depends on all kinds of factors that interact with the disjunction operator. Among such factors are clause type marking (e.g., declarative/interrogative word order) and intonation (e.g., pitch contour, prosodic phrase structure). This is illustrated in (6) below, where $\uparrow$ and $\downarrow$ indicate rising and falling pitch contours, respectively, and hyphenation and commas indicate the absence and presence of prosodic phrase breaks, respectively (examples (6b-d) are like our earlier example (1) but now each with a specific intonation pattern):

$$
\begin{array}{ll}
\text { a. } & \text { Igor speaks English-or-French } \downarrow . \\
\text { b. } & \text { Does Igor speak English-or-French } \uparrow ? \\
\text { c. } & \text { Does Igor speak English } \uparrow, \text { or French } \uparrow ? \\
\text { d. } & \text { Does Igor speak English } \uparrow, \text { or French } \downarrow \text { ? }
\end{array}
$$

While all these disjunctive sentences contain exactly the same lexical material, they each receive a different interpretation. A treatment of disjunction that takes both informative and inquisitive aspects of meaning into consideration is necessary to account for these different interpretations in a uniform way, i.e., without stipulating lexical ambiguity of the disjunction word. However, such a treatment of disjunction is not sufficient; it needs to be complemented by an account of the different clause type markers and intonational features. For an explicit account of disjunctive declaratives and interrogatives that treats disjunction uniformly as the inquisitive join operator we refer to Roelofsen and Farkas (2015).

Disjunction across languages. No matter whether we construe propositions as sets of possible worlds, as in standard truth-conditional semantics, or as downward closed sets of information states, as in inquisitive semantics, the algebraic perspective on meaning leads us to expect that many natural languages contain words or morphemes that can be used to express the basic algebraic operations on propositions, like the join operator. The inquisitive algebraic perspective on meaning gives rise to a further typological expectation as well. Namely, since the join operator in inquisitive semantics generates multiple alternatives and therefore gives rise to inquisitiveness (unlike the other basic algebraic operations, as witnessed by the examples involving conjunction and negation 
in Figure 6), it is to be expected that words which are used to express the join operation, i.e., disjunction words, may also function as question words. Interestingly, it has been observed that this is indeed the case in many languages (see, e.g., Jayaseelan, 2008; Cable, 2010; AnderBois, 2011; Slade, 2011; Szabolcsi, 2015). This is illustrated in (7) with Malayalam -oo and Japanese ka:

\begin{tabular}{lllr} 
Malayalam & Japanese & \multicolumn{2}{l}{ English translation } \\
\hline Anna-oo Peter-oo & Anna-ka Peter-ka & Anna or Peter & (disjunction) \\
Anna wannu-(w)oo & Anna wa kita-ka & Did Anna come? & (question)
\end{tabular}

AnderBois (2012) and Szabolcsi (2015) propose an account of this phenomenon in inquisitive semantics, suggesting that the inquisitive join operator can indeed be seen as the semantic common core of disjunction words and question words in languages like Malayalam and Japanese.

Hurford disjunctions. Finally, the inquisitive treatment of disjunction has repercussions for the analysis of so-called Hurford disjunctions. These are disjunctions were one disjunct implies another, giving rise to infelicity (Hurford, 1974). An example is given in (8):

(8) \#Marie lives in Paris or in France.

Such sentences have been discussed quite extensively in the recent literature (e.g., Chierchia et al., 2009; Katzir and Singh, 2013; Meyer, 2014). This line of work assumes a standard truth-conditional semantic treatment of disjunction, and provides a pragmatic explanation for the infelicity of examples like (8). The basic idea is that the stronger disjunct (in this case 'in Paris') is redundant: the weaker disjunct by itself has the same meaning as the disjunction as a whole.

However, Hurford disjunctions also appear in questions (Ciardelli and Roelofsen, 2015b):

(9) \#Does Marie live in Paris $\uparrow$, or in France $\downarrow$ ?

Assuming that in questions disjunction cannot be treated as a truth-conditional operator, but rather has to be treated as introducing multiple alternatives, the redundancy-based accounts of Hurford disjunctions cited above do not directly account for the infelicity of (9).

The question that arises, then, is how these accounts fare when re-implemented in alternative semantics or inquisitive semantics? Ideally, this would give us a uniform account of Hurford disjunctions both in declaratives and in questions. Now, interestingly, there is a difference here between the two alternative-sensitive frameworks. In inquisitive semantics, a Hurford disjunction is still predicted to be redundant: the weaker disjunct by itself has the same meaning as the disjunction as a whole. This is because propositions in inquisitive semantics are downward closed sets of states, and the alternatives in a proposition are its maximal elements. This means that one alternative can never be contained in another (otherwise it would not be a maximal element). By contrast, in alternative semantics one alternative may very well be contained in another. As a consequence, Hurford disjunctions are not predicted to be redundant in alternative semantics. In this case, the stronger disjunct makes a non-trivial contribution to the meaning of the disjunction as a whole. Thus, it seems that the inquisitive treatment of disjunction is advantageous here. For a more in-depth discussion of this issue we refer to Ciardelli and Roelofsen (2015b). 


\section{References}

Aloni, M. (2002). Free choice in modal contexts. In M. Weisgerber, editor, Proceedings of Sinn und Bedeutung. University of Konstanz.

Aloni, M. (2007a). Expressing ignorance or indifference. Modal implicatures in BiOT. In B. ten Cate and H. Zeevat, editors, Proceedings of the Sixth International Tbilisi Symposium on Language, Logic and Computation, pages 1-20, Berlin Heidelberg. Springer-Verlag.

Aloni, M. (2007b). Free choice, modals and imperatives. Natural Language Semantics, 15(1), 65-94.

Aloni, M. and Ciardelli, I. (2013). A logical account of free-choice imperatives. In M. Aloni, M. Franke, and F. Roelofsen, editors, The dynamic, inquisitive, and visionary life of $\varphi, ? \varphi$, and $\diamond \varphi$ : a festschrift for Jeroen Groenendijk, Martin Stokhof, and Frank Veltman, pages 1-17. ILLC Publications.

Aloni, M. and Égré, P. (2010). Alternative questions and knowledge attributions. The Philosophical Quarterly, 60(238), 1-27.

Aloni, M. and van Rooij, R. (2002). The dynamics of questions and focus. In B. Jackson, editor, Proceedings of SALT 12. Cornell University, CLC Publications.

Alonso-Ovalle, L. (2006). Disjunction in Alternative Semantics. Ph.D. thesis, University of Massachusetts, Amherst.

Alonso-Ovalle, L. (2009). Counterfactuals, correlatives, and disjunction. Linguistics and Philosophy, 32, 207-244.

AnderBois, S. (2011). Issues and alternatives. Ph.D. thesis, University of California Santa Cruz.

AnderBois, S. (2012). Focus and uninformativity in Yukatek Maya questions. Natural Language Semantics, 20, 349-390.

AnderBois, S. (2014). The semantics of sluicing: Beyond truth conditions. Language, 90(4), 887-926.

Bartels, C. (1999). The intonation of English statements and questions: a compositional interpretation. Routledge.

Beck, S. and Kim, S.-S. (2006). Intervention effects in alternative questions. The Journal of Comparative Germanic Linguistics, 9(3), 165-208.

Biezma, M. and Rawlins, K. (2012). Responding to alternative and polar questions. Linguistics and Philosophy, 35(5), 361-406.

Cable, S. (2010). The Grammar of Q: Q-Particles, Wh-Movement and Pied-Piping. Oxford University Press.

Champollion, L. (2015). Ten men and women got married today: Noun coordination and the intersective theory of conjunction. Journal of Semantics. 
Champollion, L., Ciardelli, I., and Zhang, L. (2015). Antecedents of counterfactuals violate de Morgans law. Talk at Leiden Utrecht Semantics Happenings (LUSH).

Chemla, E. (2009). Similarity: towards a unified account of scalar implicatures, free choice permission and presupposition projection. Manuscript, ENS Paris.

Chierchia, G., Fox, D., and Spector, B. (2009). Hurford's constraint and the theory of scalar implicatures: evidence for embedded implicatures. In Presuppositions and implicatures, pages 47-62. MIT Press Cambridge, MA.

Ciardelli, I. (2014). Question meanings = resolution conditions. Talk at Semantics and Philosophy in Europe, Berlin.

Ciardelli, I. and Roelofsen, F. (2015a). Alternatives in Montague grammar. In E. Csipak and H. Zeijlstra, editors, Proceedings of Sinn und Bedeutung, pages 161-178, Göttingen, Germany.

Ciardelli, I. and Roelofsen, F. (2015b). Hurford disjunctions and the nature of alternatives. Manuscript, University of Amsterdam.

Ciardelli, I., Groenendijk, J., and Roelofsen, F. (2013). Inquisitive semantics: a new notion of meaning. Language and Linguistics Compass, 7(9), 459-476.

Ciardelli, I., Groenendijk, J., and Roelofsen, F. (2015). Inquisitive semantics. ESSLLI lecture notes, available via www.illc.uva.nl/inquisitivesemantics.

Ellis, B., Jackson, F., and Pargetter, R. (1977). An objection to possible-world semantics for counterfactual logics. Journal of Philosophical Logic, 6(1), 355-357.

Fine, K. (1975). Critical notice on Counterfactuals by D. Lewis. Mind, 84(1), 451-458.

Fine, K. (2012). Counterfactuals without possible worlds. The Journal of Philosophy, 109(3), $221-246$.

Fox, D. (2007). Free choice disjunction and the theory of scalar implicatures. In U. Sauerland and P. Stateva, editors, Presupposition and implicature in compositional semantics, pages 71-120. Palgrave Macmillan, Basingstoke.

Franke, M. (2009). Signal to act: Game theory in pragmatics. Ph.D. thesis, University of Amsterdam.

Gazdar, G. (1980). A cross-categorial semantics for coordination. Linguistics and Philosophy, 3(3), 407-409.

Geurts, B. (2005). Entertaining alternatives: Disjunctions as modals. Natural Language Semantics, 13(4), 383-410.

Groenendijk, J. and Stokhof, M. (1984). Studies on the Semantics of Questions and the Pragmatics of Answers. Ph.D. thesis, University of Amsterdam.

Haida, A. (2010). On the semantics and pragmatics of yes/no-questions, yes/no-question disjunctions, and alternative questions: evidence from Chadic. Manuscript, Humboldt University of Berlin, draft version of October 8, 2010. 
Hamblin, C. L. (1973). Questions in Montague English. Foundations of Language, 10(1), 41-53.

Han, C.-h. and Romero, M. (2004). The syntax of whether/Q... or questions: ellipsis combined with movement. Natural Language $\mathscr{E}$ Linguistic Theory, 22(3), 527-564.

Haspelmath, M. (2007). Coordination. In T. Shopen, editor, Language typology and syntactic description, volume II: complex constructions, pages 1-51. Cambridge University Press.

Hurford, J. (1974). Exclusive or inclusive disjunction. Foundations of Language, 11(3), 409-411.

Jayaseelan, K. (2008). Question particles and disjunction. Manuscript, The English and Foreign Languages University, Hyderabad.

Karttunen, L. (1977). Syntax and semantics of questions. Linguistics and Philosophy, 1, 3-44.

Katzir, R. and Singh, R. (2013). Hurford disjunctions: embedded exhaustification and structural economy. In Proceedings of Sinn und Bedeuting 18, pages 201-216.

Keenan, E. L. and Faltz, L. M. (1985). Boolean semantics for natural language. Springer.

Klinedinst, N. (2009). (Simplification of $)^{2}$ disjunctive antecedents. MIT Working Papers in Linguistics, 60.

Kratzer, A. (2005). Indefinites and the operators they depend on: From Japanese to Salish. Reference and quantification: The Partee effect, pages 113-142.

Kratzer, A. and Shimoyama, J. (2002). Indeterminate pronouns: The view from Japanese. In Y. Otsu, editor, The Proceedings of the Third Tokyo Conference on Psycholinguistics, pages $1-25$.

Lewis, D. (1973). Counterfactuals. Blackwell.

Menéndez-Benito, P. (2005). The grammar of choice. Ph.D. thesis, University of Massachusetts, Amherst.

Meyer, M.-C. (2014). Deriving Hurford's constraint. In M. W. Todd Snider, Sarah D'Antonio, editor, Semantics and Linguistic Theory (SALT), volume 24, pages 577-596. LSA and CLC Publications.

Nute, D. (1975). Counterfactuals and the similarity of words. The Journal of Philosophy, 72(21), $773-778$.

Partee, B. H. and Rooth, M. (1983). Generalized conjunction and type ambiguity. In R. Bäuerle, C. Schwarze, and A. von Stechow, editors, Meaning, Use and Interpretation of Language, pages 115-143. De Gruyter, Berlin.

Pruitt, K. and Roelofsen, F. (2011). Disjunctive questions: prosody, syntax, and semantics. Presented at a seminar at the Georg August Universität Göttingen. Available via www.illc.uva.nl/inquisitivesemantics.

Pruitt, K. and Roelofsen, F. (2013). The interpretation of prosody in disjunctive questions. Linguistic Inquiry, 44, 632-650. 
Rawlins, K. (2013). (Un)conditionals. Natural language semantics, 21(2), 111-178.

Roelofsen, F. (2013a). Algebraic foundations for the semantic treatment of inquisitive content. Synthese, 190(1), 79-102.

Roelofsen, F. (2013b). An inquisitive perspective on meaning: the case of disjunction. Stanford Linguistics Colloquium.

Roelofsen, F. and Farkas, D. F. (2015). Polarity particle responses as a window onto the interpretation of questions and assertions. Language, 91(2), 359-414.

Roelofsen, F. and van Gool, S. (2010). Disjunctive questions, intonation, and highlighting. In M. Aloni, H. Bastiaanse, T. de Jager, and K. Schulz, editors, Logic, Language, and Meaning: Selected Papers from the Seventeenth Amsterdam Colloquium, pages 384-394. Springer.

van Rooij, R. (2006). Free choice counterfactual donkeys. Journal of Semantics, 23(4), 383-402.

van Rooij, R. (2010). Conjunctive interpretations of disjunctions. Semantics and Pragmatics, $\mathbf{3}(11), 1-28$.

Rooth, M. (1985). Association with Focus. Ph.D. thesis, University of Massachusetts, Amherst.

Schulz, K. (2005). A pragmatic solution for the paradox of free choice permission. Natural Language Semantics, 13(3), 271-316.

Simons, M. (2005a). Dividing things up: the semantics of or and the modal/or interaction. Natural Language Semantics, 13(3), 271-316.

Simons, M. (2005b). Semantics and pragmatics in the interpretation of or. In E. Georgala and J. Howell, editors, Proceedings of Semantics and Linguistic Theory (SALT 15), pages 205-222. Cornell University, CLC Publications.

Slade, B. (2011). Formal and philological inquiries into the nature of interrogatives, indefinites, disjunction, and focus in Sinhala and other languages. Ph.D. thesis, University of Illinois, UrbanaChampaign.

Stalnaker, R. (1968). A theory of conditionals. In N. Rescher, editor, Studies in Logical Theory. Blackwell, Oxford.

Stalnaker, R. (1978). Assertion. Syntax and Semantics, 9, 315-332.

von Stechow, A. (1991). Focusing and backgrounding operators. In W. Abraham, editor, Discourse Particles: descriptive and theoretical investigations on the logical, syntactic and pragmatic properties of discourse particles in German, pages 37-84. John Benjamins, Amsterdam.

Szabolcsi, A. (2015). What do quantifier particles do? Linguistics and Philosophy, 38(2), 159-204.

Theiler, N. (2014). A multitude of answers: embedded questions in typed inquisitive semantics. MSc thesis, University of Amsterdam.

Uegaki, W. (2014). Japanese alternative questions are disjunctions of polar questions. In Proceedings of SALT 24. 
Warmbrōd, K. (1981). Counterfactuals and substitution of equivalent antecedents. Journal of Philosophical Logic, 10(2), 267-289.

Zimmermann, E. (2000). Free choice disjunction and epistemic possibility. Natural Language Semantics, 8, 255-290. 